\title{
Bovine tuberculosis breakdown duration in cattle herds: an investigation of herd, host, pathogen and wildlife risk factors
}

\author{
Georgina Milne ${ }^{\text {Corresp., } 1}$, Adrian Allen ${ }^{1}$, Jordon Graham ${ }^{1}$, Angela Lahuerta-Marin ${ }^{1}$, Carl McCormick ${ }^{1,2}$, Eleanor \\ Presho $^{1}$, Neil Reid ${ }^{3}$, Robin Skuce ${ }^{1}$, Andrew W Byrne ${ }^{\text {Corresp. 1, 4, } 5}$ \\ ${ }^{1}$ Veterinary Sciences Division, Agri-food and Biosciences Institute, Belfast, United Kingdom \\ 2 Department of Agriculture, Environment, and Rural Affairs, Coleraine, United Kingdom \\ 3 School of Biological Sciences, Queen's University Belfast, Belfast, United Kingdom \\ 4 One-Health Scientific Support Unit, Department of Agriculture, Food and the Marine, Dublin, Ireland \\ 5 School of Biological Sciences, Queens University Belfast, Belfast, United Kingdom \\ Corresponding Authors: Georgina Milne, Andrew W Byrne \\ Email address: georgina.milne@afbini.gov.uk, andreww.byrne@agriculture.gov.ie
}

Background. Despite rigorous controls placed on herds which disclose antemortem test positive cattle to bovine tuberculosis, caused by the infection of Mycobacterium bovis, many herds in Northern Ireland (NI) experience prolonged breakdowns. These herds represent a considerable administrative and financial burden to the State and farming community.

Methods. A retrospective observational study was conducted to better understand the factors associated with breakdown duration, which was modelled using both negative binomial and ordinal regression approaches.

Results: Six explanatory variables were important predictors of breakdown length in both models; herd size, the number of reactors testing positive in the initial SICCT test, the presence of a lesioned animal at routine slaughter (LRS), the count of M. bovis genotypes during the breakdown (MLVA richness), the local herd-level bTB prevalence, and the presence of herds linked via management factors (associated herds). We report that between 2008 and 2014, mean breakdown duration in NI was 226 days (approx. seven months; median; 188 days). In the same period, however, more than $6 \%$ of herds in the region remained under movement restriction for more than 420 days (13 months); almost twice as long as the mean. The MLVA richness variable was a particularly important predictor of breakdown duration. We contend that this variable primarily represents a proxy for beef fattening herds, which can operate by purchasing cattle and selling animals straight to slaughter, despite prolonged trading restrictions. For other herd types, the model supports the hypothesis that prolonged breakdowns are a function of both residual infection within the herd, and infection from the environment (e.g. infected wildlife, contiguous herds and/or a contaminated environment). The impact of badger density on breakdown duration was assessed by including data on main sett (burrow) density. Whilst a positive association was observed in the univariate analysis, confounding with other variables means that the contribution of badgers to prolonged breakdowns was not clear from our study. We do not fully reject the hypothesis that badgers are implicated in prolonging bTB breakdowns via spillback infection, but given our results, we posit that increased disease risk from badgers is unlikely to simply be a function of increasing badger density measured using sett metrics. 
Bovine tuberculosis breakdown duration in cattle herds: an investigation of herd, host, pathogen and wildlife risk factors

Margaret Georgina Milne ${ }^{1}$, Adrian Allen ${ }^{1}$, Jordon Graham ${ }^{1}$, Angela Lahuerta-Marin ${ }^{1}$, Carl

McCormick $^{1 \dagger}$, Eleanor Presho ${ }^{1}$, Neil Reid ${ }^{2}$, Robin Skuce ${ }^{1,2}$, Andrew William Byrne ${ }^{1,2 \wedge}$

${ }^{1}$ Veterinary Sciences Division, Agri-food and Biosciences Institute, Belfast, United Kingdom

${ }^{2}$ School of Biological Sciences, Queen’s University Belfast, Belfast, United Kingdom

† Current address: Department of Agriculture, Environment, and Rural Affairs, Coleraine, United Kingdom

${ }^{\wedge}$ Current address: One-Health Scientific Support Unit, Department of Agriculture, Food and the Marine, Dublin, Ireland

Corresponding Author:

Dr. Georgina Milne \& Dr. Andrew Byrne

Veterinary Sciences Division, Agri-food and Biosciences Institute (AFBI),

12 Stoney Road, Stormont, Belfast BT4 3SD, UK Email address: georgina.milne@afbini.gov.uk; ecologicalepidemiology@gmail.com 


\section{Abstract}

\section{Background.}

3 Despite rigorous controls placed on herds which disclose antemortem test positive cattle to

4 bovine tuberculosis, caused by the infection of Mycobacterium bovis, many herds in Northern

5 Ireland (NI) experience prolonged breakdowns. These herds represent a considerable

6 administrative and financial burden to the State and farming community.

7 Methods. A retrospective observational study was conducted to better understand the factors

8 associated with breakdown duration, which was modelled using both negative binomial and

9 ordinal regression approaches.

Results. Six explanatory variables were important predictors of breakdown length in both models; herd size, the number of reactors testing positive in the initial SICCT test, the presence

12 of a lesioned animal at routine slaughter (LRS), the count of $M$. bovis genotypes during the breakdown (MLVA richness), the local herd-level bTB prevalence, and the presence of herds

14 linked via management factors (associated herds).

15 We report that between 2008 and 2014, mean breakdown duration in NI was 226 days (approx. 16 seven months; median; 188 days). In the same period, however, more than $6 \%$ of herds in the 17 region remained under movement restriction for more than 420 days (13 months); almost twice 18 as long as the mean. The MLVA richness variable was a particularly important predictor of

19 breakdown duration. We contend that this variable primarily represents a proxy for beef fattening

20 herds, which can operate by purchasing cattle and selling animals straight to slaughter, despite 21 prolonged trading restrictions. For other herd types, the model supports the hypothesis that 
22 prolonged breakdowns are a function of both residual infection within the herd, and infection

23 from the environment (e.g. infected wildlife, contiguous herds and/or a contaminated

24 environment). The impact of badger density on breakdown duration was assessed by including

25 data on main sett (burrow) density. Whilst a positive association was observed in the univariate

26 analysis, confounding with other variables means that the contribution of badgers to prolonged

27 breakdowns was not clear from our study. We do not fully reject the hypothesis that badgers are 28 implicated in prolonging bTB breakdowns via spillback infection, but given our results, we posit

29 that increased disease risk from badgers is unlikely to simply be a function of increasing badger 30 density measured using sett metrics.

31

32 


\section{Introduction}

34

35

36

37

Bovine tuberculosis (bTB), caused by Mycobacterium bovis bacterial infection, presents an ongoing epidemic in many countries (Humblet et al. 2009). In Britain and Ireland, bTB remains stubbornly persistent despite long-term and intensive programs focusing primarily on controlling bTB breakdowns in cattle herds (Allen et al. 2018). In Northern Ireland (NI) infection levels remain high, with an annual herd level incidence of over 8\% (DAERA 2018). Current bTB controls are compliant with EU Directive 64/432/EEC (as amended) and consist of a "test-and-slaughter policy", alongside active routine slaughterhouse surveillance (Abernethy et al. 2006; Abernethy et al. 2013). Herds undergo annual testing using the single intradermal comparative cervical tuberculin (SICCT) test, with infected herds subsequently placed under trading restrictions (a herd breakdown) until two clear herd level tests are obtained, each not less than 60 days apart (DAERA 2017). The shortest length of time a herd usually remains under trading restriction is therefore 120 days. All animals which test positive to the SICCT test are culled. To aid in the detection and eradication of bTB, additional testing can be undertaken in persistently infected herds using the interferon gamma test (Lahuerta-Marin et al. 2015). Despite these efforts, some herds fail to clear infection upon retest and remain persistently infected, resulting in prolonged or recurrent breakdowns (Doyle et al. 2016; Milne et al. 2019). As herdkeepers are compensated for culled cattle, the remuneration costs of persistent breakdowns contribute disproportionally to the total program costs, which have exceeded $£ 30$ million per annum in recent years (NIAO 2018). Additionally, the trading restrictions and production losses associated with persistent breakdowns also present considerable economic and emotional burdens to the farming community (Robinson 2017). 

these persistently infected (i.e. "chronic") herds using a number of non-mutually exclusive

58

59

60

61 62

1

criteria. These include recurrence of bTB in a herd (Doyle et al. 2016; Gallagher et al. 2013;

Karolemeas et al. 2012; Karolemeas et al. 2011; Wolfe et al. 2010), the prolongation of trading restrictions (Doyle et al. 2016; Griffin et al. 1993; Karolemeas et al. 2012; Karolemeas et al. 2010) and outbreak size (Clegg et al. 2018). Here, we focus specifically on bTB persistence as defined by breakdown length and measured via the duration of movement restriction periods.

Indeed, extended periods of trading restrictions have been observed in herds across these islands (More et al. 2018). For example, in the ROI in 2012, 16.8\% of herds were restricted for over 255 days (seven and a half months) (Houtsma et al. 2018). In England, 5.8\% of breakdowns in England lasted longer than 550 days (18 months), in contrast to the mean breakdown length of 192 days (AHVLA 2016). In NI, a previous study found that the median breakdown duration was 184 days (approx. six months) (Doyle et al. 2016), however between 2003 and 2015, 4.3\% of bTB breakdowns in NI were classified as prolonged ( $>550$ days) (More et al. 2018).

Earlier work has enabled better understanding of the factors associated with breakdown duration. A previous study from NI between 2005 and 2010 showed that bTB breakdowns lasting longer than 365 days were associated with local area bTB prevalence, the presence of associated herds (i.e. herds linked via geography, family or some other management factor), the number of years previously restricted, the number of cattle reactors at the disclosing test, the total number of reactors over the outbreak, and the identification of a lesion consistent with bTB at routine slaughter (Doyle et al. 2016). In Great Britain (GB) prolonged breakdowns were particularly associated with the confirmation status of the breakdown and herd size (Karolemeas 
79 et al. 2010). Comparisons between transient bTB breakdowns ( $\leq 6$ months) and breakdowns

80 lasting $>6$ months (i.e. "persistent") in GB found that herd size, herd management, and the

81 presence of active badger setts were important explanatory variables associated with bTB

82 persistence (Reilly \& Courtenay 2007). Whilst breakdown prolongation is either a feature of

83 failure to clear infection from the herd (i.e. within herd recrudescence), and/or re-infection from

84 local sources (e.g. contagious herds or a local wildlife reservoir), it is not yet possible to

85 disentangle these various routes of infection.

86

87

The European badger (Meles meles) is a well-documented infection reservoir for M. bovis (Byrne et al. 2014b; Gallagher \& Clifton-Hadley 2000) and a number of other studies have also explored the association between bTB breakdown duration and wildlife. In the ROI, the presence of badgers was associated with bTB breakdowns lasting greater than one year (Griffin et al. 1993), and reactive badger culling was related to the prolongation of bTB breakdowns in GB (Karolemeas et al. 2012). However, the contribution that badgers make towards protracted bTB breakdowns is not well understood to date. Furthermore, previous work largely consists of case-control studies, and does not model breakdown length explicitly. The aim of this work, therefore, was to model the factors associated with breakdown duration, including variables associated with M. bovis molecular genotype data (Skuce et al. 2010), badger density data (Reid

97 et al. 2012), alongside herd characteristics. For the first time, breakdown length was modelled as both a continuous and ordinal variable, which we believe improves our understanding of withinherd bTB dynamics and could be applied to bTB management in many endemic regions globally. 
102 Study Area

103 Northern Ireland is approximately $14,000 \mathrm{~km}^{2}$. The official bTB control programme is 104 administered over ten Divisional Veterinary Office DVO areas, comprised of 123 "patches"; 105 mean size $110 \mathrm{~km}^{2}(\mathrm{SD} \pm 53)$; Fig. 1.

106

107 Study design

108 Two retrospective analyses were undertaken, firstly (i) quantifying the risk factors associated with bTB breakdown duration, using negative binomial (count) regression with the

110 outcome measured in days, and (ii) quantifying the risk associated with bTB breakdown duration

111 using ordinal regression, with the outcome modelled as a categorical ordered variable. This

112 approach was considered necessary to account for bTB breakdown administration in NI. Herd

113 breakdown duration measures arise as a result of a disease management process, and are not a

114 wholly natural phenomenon. Generally, once bTB has been confirmed, the herd Officially

115 Tuberculosis free status is Withdrawn (OTW). Usually, two clear herd tests are required to

116 restore Officially Tuberculosis Free (OTF) status. Each herd-level test is scheduled to occur a

117 minimum of 60 days apart (DAERA 2018a). For the ordinal regression therefore, breakdown

118 duration was classified into four distinct categories based on multiples of 60 days (it should be

119 noted, however, that breakdown length may not always correlate exactly with the number of tests

120 done, as herds may delay testing). The first category contained breakdowns $\leq 180$ days (approx.

1216 months; 3 tests until OTF status restored), the second category included breakdowns which

122 ended up to 120 days later; $\leq 300$ days (approx. 9 months; 5 tests until OTF status restored), and

123 the third category included breakdowns which ended up to 120 days after this; $\leq 420$ days

124 (approx. 13 months; 7 tests until OTF status restored). The final category included breakdowns 
125 which lasted longer than 421 days ( 8 or more tests until OTF status restored). Breakdown start

126 dates are denoted by the date at which the first SICCT reactor or lesioned animal identified at

127 slaughter was disclosed, and the breakdown end date was the test at which the last clear herd test

128 was achieved.

129

130 Dataset creation

131 BTB breakdown data spanning January 2003 to December 2015 inclusive (n breakdowns

$132=27,718$ ) were made available from the NI Department of Agriculture, Environment and Rural

133 Affairs (DAERA) database, the Animal and Public Health Information System (APHIS)

134 (Houston 2001). This dataset was restricted to only include OTW breakdowns ( $\mathrm{n}=19,084$;

1358,634 breakdowns removed), which were defined by policy guidance at the time of study as the 136 presence of more than five SICCT reactors, or two positive results to the four possible bTB tests;

137 confirmation via histopathology, culture or spoligotyping, or the identification of a lesion at

138 routine slaughter. Breakdowns with incomplete or erroneous information were also excluded

139 (e.g. missing GIS information, MLVA information, or breakdowns lacking end dates $(\mathrm{n}=$

14017,$114 ; 1,970$ breakdowns removed). The dataset was further restricted to include breakdowns

141 which started and ended between $01 / 01 / 2009$ and 31/12/2014 ( $\mathrm{n}=7,478 ; 9,636$ breakdowns

142 removed). These dates were chosen because surveillance using M. bovis MLVA genotyping data

143 occurred at the herd level between 2003 and 2008, but from 2009 onwards, all culture confirmed

144 animal-level M. bovis isolates were genotyped. Finally, breakdowns which were recorded as

145 lasting less than 60 days were excluded from the final dataset ( $\mathrm{n}=5$ breakdowns removed), as 60

146 days is the minimum restriction period which may be permitted under some circumstances e.g.

147 less than five positive SICCT animals with no post-mortem or laboratory confirmation (DAERA 
148 2019). The final dataset contained information on 7,473 breakdowns. All data were assembled

149 and analysed using Microsoft Access 2007 (12.0.6735.5000) SP3 MSO and R Version 3.2.5 (R

150 Core Team, 2013).

151

152

The fixed-effect variables considered in the analysis are shown in Table 1. They were

153

154

155

156

157

158

159

160

161

162

163

164

165

166

167

168

169

170 derived and defined as follows; herd size (number of animals in the herd at the time of

breakdown); outbreak reactors (the number of SICCT reactors present in the disclosing test);

total reactors (the total number of SICCT reactors during a breakdown); yearly patch prevalence (herd level bTB prevalence for the year); mean patch prevalence (mean herd-level bTB

prevalence), outward moves year before (the number of outward cattle moves in the year prior to breakdown), and inward moves year before (the number of inward cattle moves in the year prior to breakdown). A categorical herd type variable was included (beef, dairy, other, or unknown). Binary variables were the presence or absence of a milk license, whether lesions consistent with tuberculosis were identified during routine slaughter (LRS), the presence or absence of associated herds (herds are "associated" via e.g. shared management, shared grazing, or shared family responsibilities), and whether the herd had any previous breakdowns during the study period. The herd DVO, the year of breakdown, and the herd unique identifier were included as random effect variables. The distribution of explanatory variables across each DVO in NI is illustrated in Fig. 1.

\section{M. bovis MLVA genotype data}

M. bovis MLVA genotype data were derived from isolates obtained from skin-test reactors, and from lesioned animals identified at routine slaughter. These animal-level data were 
171 then associated with bTB breakdown-level data. From this, breakdown-level metrics of MLVA

172 genotype richness (number of different MLVA types) were calculated. The process of

173 genotyping M. bovis isolates has been described more fully elsewhere (Kamerbeek et al. 1997;

174 Skuce et al. 2010; Skuce et al. 2005). Briefly, all culture-confirmed bTB cases were sub-

175 cultured to single colonies and heat-killed to create PCR-ready bacterial cell lysates. These were

176 then used as PCR templates for molecular characterisation of pathogen variation. Eight VNTR

177 loci across the M. bovis genome were genotyped; MV2163B/QUB11B, MV4052/QUB26A,

178 MV2461/ETRB, MV1955/Mtub21, MV1895/QUB1895, MV2165/ETRA, MV2163/QUB11A

179 and MV3232/QUB3232 (Durr et al. 2000).

180

181 Badger density

182

Badger main sett density was incorporated into models by using a data from the Northern

Ireland Badger Survey 2007-08 (Reid et al. 2012). This enumerated and mapped badger main setts

within 212 regularly spaced $1 \mathrm{~km}^{2}$ squares throughout Northern Ireland, and subsequently spatially

interpolated using the Kriging function of the ArcMap 10.5 (ESRI, California, USA) Spatial

Analyst toolbox providing a heat-map proxy of badger density throughout the region (Reid et al. 2012).

Data modelling

During the univariable stage of model fitting for both the count and ordinal models,

predictor variables were explored using summary statistics and cross-tabulations with the scrutinised using ggplot2 (Wickham 2009). Predictor variables were then considered 
194 individually for association with the outcome. Correlation coefficients between variables were

195 determined. Variables with moderate or strong correlation $\geq 0.5$ or $\leq-0.5$ were identified, and

196 from these, only those variables with the strongest association with the outcome were retained,

197 based on log-likelihood values. Following univariable assessment, generalised linear mixed

198 models (GLMMs) were fitted. The count model was constructed using the package lme4 (Bates

199 et al., 2015), and the ordinal model was constructed using the package ordinal (Christensen

200 2019). Initial modelling of the count data using Poisson regression indicated the presence of

201 over-dispersion (the variance was greater than the mean); a negative binomial model was instead

202 found to be more suitable for these data (Zuur et al. 2015; Zuur 2009)

203

204 In both count and ordinal models, the DVO, breakdown year, and herd identifier were

205 included as nested random effects (Zuur 2009). Continuous variables were log-transformed in

206 the final models for computational efficiency, and to improve the model fit (i.e. ensure all

207 explanatory variables were on the same scale, to approximate a more linear relationship,

208 reducing skew and to limit the influence of outliers). All predictors were initially included in the

209 model, including biologically plausible two-way interactions. Final models were assembled

210 using backwards stepwise selection routines; better fitting models were selected on the basis of

211 likelihood ratio tests (Christensen 2019; Zuur 2009). At each stage, however, model coefficients

212 were manually assessed for confounding (Dohoo et al., 2009). Once final models were

213 constructed, excluded predictor variables were again offered to the model and the impact

214 assessed using likelihood ratio tests. Final models were screened for correlations between fixed

215 effects and random effects and were assessed by visual examination of residuals. Plots of

216 residual versus fitted values were firstly explored; residuals were then plotted against all 
217 covariates included in the model, and also against the covariates which had been excluded during

218 model fitting. Residuals were used to identify influential data-points, and models were re-run

219 with these data removed for comparative purposes.

220

221

Ordinal regression assumes that the effects of explanatory variables are consistent across

222 all outcome categories (i.e. the assumption of proportional odds). We firstly attempted to test

223 this using the nominal_test function of the Ordinal package (Christensen 2019). However, at the

224 time of analysis, this function was not available for models with multiple random effects.

225 Furthermore, it is presently not feasible to construct an ordered regression model with multiple

226

random effects for which the assumption of proportional odds is also relaxed (Christensen 2019).

227 To overcome this, we constructed an initial ordered regression model including only fixed effects

228 (via the $\mathrm{clm}$ function) and tested the assumption of proportional odds on this model (the

229 nominal_test function). Explanatory variables which violated the assumption of proportional

230 odds were identified and the model was re-ran, wherein the proportional odds assumption was

231 relaxed for these variables. However, the final $\mathrm{clmm}$ model was further validated by comparing

232 the model coefficients against those derived from three binary logistic GLMMs (Armstrong \&

233 Sloan 1989) (Ananth \& Kleinbaum 1997), with the binary outcome variable dichotomised at the

234 same levels as in the ordinal regression. In these three models, the outcome (breakdown length)

235 was dichotomised as follows: Model 1; $\leq 180$ days (breakdowns 180 days or less classified as 0 ,

236 all others classified as 1); Model $2 \leq 300$ days; (breakdowns 300 days or less classified as 0, all

237 others classified as 1); and Model $3 \leq 420$ days (breakdowns 420 days or less classified as 0 , all

238 others classified as 1). 


\section{Results}

\section{Summary data}

243 breakdown length was 226 days ( $\mathrm{SD} \pm 140$ days; approx. seven months) and median breakdown 244 length was 188 days (Inter Quartile Range (IQR): 140-260 days; approx. six months). The 245 longest breakdown was recorded at 2,288 days (6 years). When classified into categories, almost 246 half of all breakdowns $(47.18 \%, \mathrm{n}=3,526)$ lasted less than 180 days. $34.86 \%(\mathrm{n}=2,605)$ of 247 breakdowns were between 181 and 300 days in duration, 11.33\% $(\mathrm{n}=847)$ lasted between 301 248 and 420 days, whilst $6.62 \%$ of all bTB breakdowns $(n=495)$ lasted 421 days or longer $(13$ 249 months; i.e. 8 or more tests were required to restore OTF status). The distribution of the 250 breakdown length outcome variable is shown in Fig. 2A-B. Mean breakdown duration varied 251 across NI, from a minimum of 192 days in Derry/Londonderry DVO to a maximum of 266 days 252 in Newry DVO (Fig. 1).

253

254

Count model results

255 The results of the count model of breakdown duration is shown in Table 2 (Table S1).

256 The final model contained seven explanatory variables. The exponentiated results are reported

257 here as Incidence Rate Ratios (IRR) with associated 95\% upper and lower confidence intervals 258 (CI). The variables $\log$ herd size (IRR: 1.05, 95\%CI: 1.04-1.06), $\log$ outbreak reactors (IRR: 259 1.05, 95\%CI: 1.04-1.06), $\log$ mean patch prevalence (IRR: 1.04, 95\%CI: 1.01-1.07) and $\log$ 260 MLVA richness (IRR: 1.62, 95\%CI: 1.58-1.67) were positively associated with breakdown 261 duration. The binary variables for presence of an LRS (IRR: 1.12, 95\%CI: 1.09-1.14), presence 262 of associated herds (IRR: 1.10, 95\%CI: 1.07-1.13) and a previous breakdown (IRR: 1.04, 
263 95\%CI: 1.02-1.07) were positively associated with breakdown duration. Re-running the model 264 with influential data removed resulted in only minimal change in parameter estimates when 265 compared to the original model ( $<15 \%$ change). The addition of a quadratic term for log MLVA 266 richness was also found to significantly lower log-likelihood; this model is shown in Table S2.

267

268

269

270

271

272

273

274

275

276

277

278

279

280

281

282

283

284

285

\section{Ordinal model results}

Six variables were identified as important predictors in the ordinal model. The parameter estimates of the final model are shown in Table 2 (Table S3). All six variables in the final model were found to be positively associated with the increasing breakdown duration; log herd size (OR: 1.26, 95\%CI: 1.20-1.32), $\log$ outbreak reactors (OR: 1.34, 95\%CI: 1.26-1.43), $\log$ mean patch prevalence (OR: $1.20,95 \% \mathrm{CI}: 1.04-1.37), \log$ MLVA richness (OR: 7.06, 95\%CI: 6.048.24), the presence of an LRS (OR: 1.79, 95\%CI: 1.59-2.01) and the presence of associated herds (OR: 1.49, 95\%CI: 1.32-1.69). The coefficients derived from this model were similar to a fixedeffect ordinal regression model, however the variables $\log$ herd size, $\log$ MLVA richness and $\log$ outbreak reactors violated the proportional odds assumption $(\mathrm{p}<0.05)$, suggesting that the effect size is not the same across all three breakdown duration categories. As the assumption of proportional odds was not met for all variables, the coefficients from ordinal model were also compared to those derived from three binomial logistic GLMMs (Fig. 3). There was only limited evidence of the parameter estimates differing between ordinal and binomial models. The binomial model of breakdowns lasting 420 days or less returned a higher odds ratio associated with herd size (OR: $1.49,95 \% \mathrm{CI}$ : $1.32-1.67)$ than the ordinal model (OR: $1.26,95 \% \mathrm{CI}: 1.20$ 1.32). The parameter estimate for the number of outbreak reactors was elevated in the binomial model of breakdowns lasting less than 180 days (OR: $1.56,95 \%$ CI: $1.45-1.49$ ) compared to the 
286 ordinal model (OR: 1.34, 95\%CI: 1.26-1.43), and was also diminished in the model of

287 breakdowns lasting 301 days or more (OR: 1.23, 95\%CI: 1.03-1.23) and 421 days or more (OR:

288 1.08, 95\%CI: 0.94-1.24).

289

290

MLVA Genotype richness

291

MLVA genotype richness was the most important variable in both count and ordinal

292

models, in terms of both effect size and decrease in model deviance. This was particularly

293

observable in the ordinal regression model (Fig. 4A). The MLVA genotype richness variable

294

was moderately correlated with the number of inwards moves in the year prior to breakdown $(\mathrm{r}=$

295

$0.33)$, outwards moves in the year prior to breakdown $(\mathrm{r}=0.34)$ and the number of total reactors

296

over the breakdown $(\mathrm{r}=0.39)$. Further investigation into this "total reactors" variable revealed

297 significantly more reactors in herds with a milk license $($ mean $=11)$ than herds without a milk 298 license (mean =6; Univariable Negative Binomial Regression, IRR: 1.7; 95\%CI: 1.62-1.79; Fig. 4B). However, the presence of a milk license was only 'marginally significant' in a univariable analysis of breakdown length in both count (IRR: $1.02,95 \% \mathrm{CI}$ : $1.00-1.05)$ and ordinal models 301 (OR: 1.16, 95\%CI: 1.01-1.27), and was not retained as a predictor of breakdown length in the 302 finals GLMMs after model building. Further analysis showed that whilst mean breakdown 303 length in herds with a milk license was indeed marginally longer (230 days \pm 141 ) than in herds 304 without a milk license (224 days \pm 140 ), some of the longest breakdowns were found in herds 305 without milk licenses. For example, there were 27 breakdowns lasting over 1000 days; 10 were 306 in herds with milk licenses, and 17 were in herds without; Fig. 4C. It would therefore appear 307 that whilst production type per-se is not a useful predictor of breakdown length, the results show 308 that some variables which vary between production types - the number of reactors over a 
309 breakdown for example (here, confounded with MLVA genotype richness, Fig. 4D), are indeed 310 important predictors of breakdown length.

311

312 Badger density results

When modelled using a univariate negative binomial GLM, badger main sett density was

314 a significant predictor of breakdown length (IRR: 1.13, 95\%CI: 1.13-1.14). However, this

315 variable was not retained in the final GLMM. Further investigation found that main sett density

316 was correlated with other explanatory variables. Thus, main sett density per-DVO was

317 moderately correlated with breakdown length per-DVO $(r=0.57)$ and with breakdown length

318 per-patch $(r=0.32)$, suggesting that the spatial variables already included in the model, notably

319 DVO, captured the general positive relationship observed between main sett density and

320 breakdown length; Fig. 5A. Furthermore, when compared to a fixed effects univariate GLMs

321 where DVO was the sole predictor of breakdown length, the addition of the main sett density

322 variable did not result in a better fitting model $\left(\chi^{2}=0.02, \mathrm{df}=1, p=0.90\right)$. An interaction

323 between DVO and sett density was, however, significant when compared to the fixed-effects

324 model with non-interacting DVO and main sett variables $\left(\chi^{2}=24.24, \mathrm{df}=9, p=0.004\right.$; Table S4

325 and table S5), suggesting a differential relationship between sett density and breakdown length

326 on a per-DVO basis which was not immediately observable when data were not stratified by

327 DVO. Fig. 5C-D illustrates this observation. Whilst a positive association was found between

328 main sett density and breakdown length in Ballymena, Coleraine, Dungannon, Larne and

329 Derry/Londonderry DVOs, a negative relationship between sett density and breakdown length

330 was observed in Armagh, Enniskillen, Newry, Newtownards and Omagh DVOs (Table S6). To

331 explore this further, we therefore present a second GLMM, (Table 3) in which main sett density 
332 was permitted to differ on a per-DVO basis (i.e. a random slopes and random intercepts model).

333 It should be noted, however, that the inclusion of the random slopes term for main sett resulted in

334 only marginally improvements, compared to the original GLMM (Table 2) which did not include

335 a random slope for main sett density per-DVO $\left(\chi^{2}=4.61, \mathrm{df}=2, p=0.099\right)$

336

337

338

339

340

341

342

343

344

345

346

347

348

349

350

351

352

353

354

Further analysis also indicated that that the main sett density variable exhibited moderate correlation with mean patch prevalence $(\mathrm{r}=0.40$; Fig. $3 \mathrm{C})$. To better understand the effect of main sett density on breakdown duration in the absence of spatial confounders, two further alternative models were constructed, both omitting DVO from the random effects component and including $\log$ main sett in the fixed effects component. These models also incorporated the other fixed-effect variables reported in Table 2, however, one of these models included patch prevalence in the fixed component, and the other did not. In the model which omitted both DVO and $\log$ patch prevalence, $\log$ main sett was a significant predictor of breakdown length (OR: 1.08, 95\%CI: 1.05-1.11; Table S7). Log main sett was also found to be an important predictor of breakdown length when $\log$ patch prevalence was included (OR: 1.08, 95\%CI: 1.05-1.11; Table S8), however in this model, $\log$ patch prevalence was no longer an important predictor of breakdown duration (OR: 1.01, 95\%CI: 0.99- 1.11; $\left.\chi^{2}=1.02, \mathrm{df}=1, p=0.31\right)$. Confounding between main sett density and DVO was also observed in the ordinal regression. Thus, main sett density was positively associated with increasing breakdown duration categories in a univariable GLM (OR: $1.59,95 \%$ CI: 1.42-1.78), but the main sett variable was not recovered as an important predictor of breakdown length in the mixed model context. We constructed a univariable ordinal GLM with DVO as the sole predictor of breakdown duration category. The coefficients from this model (i.e. the "risk" associated with each DVO) was positively associated 
355 with mean sett density per DVO $(r=0.59)$. Additionally, the inclusion of the main sett variable

356 in this model did not improve model fit $\left(\chi^{2}=0.04, \mathrm{df}=1, p=0.84\right)$.

357

358 Discussion

359 The heterogeneity in transmission of infections across populations is a well-known

360 phenomenon in many systems (Woolhouse et al. 1997), where a small proportion of the

361 population can contribute disproportionately to disease maintenance. Our work highlights this

362 issue in the context of prolonged bTB breakdowns. The results show that mean breakdown

363 length was 226 days (seven and a half months), and the median was 188 days (six months).

364 However, over $6 \%$ of breakdowns in this study lasted over 420 days (13 months, representative

365 of 7 herd-level tests, each 60 days apart, before OTF status was restored). Six variables

366 associated with increasing breakdown length in cattle herds in NI were identified in both models.

367 These can be grouped into three main categories; (1) variables related to herd characteristics,

368 namely herd size and herd type; (2) variables related to undetected residual infection (i.e.

369 infection within-herd), and (3) variables relating to local factors (i.e. infected wildlife, infected

370 contiguous herds and a contaminated environment,).

371

372 The MLVA genotype richness variable exhibited the strongest association with

373 breakdown duration, both regarding effect size and in contribution to model fit. Previous work

374 found that in a small number of herds, likely to be beef fattening enterprises, MLVA genotype

375 accumulation was associated with the inwards purchase of cattle from over a wide geographical

376 extent (Milne et al. 2019b). Despite this, we did not find that the number of inwards movements

377 prior to breakdown was a particularly important predictor of breakdown length, e.g. (Reilly \& 
378 Courtenay 2007). However, we did not consider inwards cattle movements during a bTB

379 breakdown, as businesses can be required to limit purchasing of cattle whilst bTB restricted, or

380 where testing delays occur, banned from purchasing (a consequence of the bTB control

381 program). Nevertheless, some beef fattening herds may indeed continue to purchase cattle

382 despite the presence of bTB, as such enterprises operate by selling animals straight to slaughter

383 (as opposed to onwards to other herds) and are only minimally impacted by movement

384 restrictions. It is therefore likely that both the elevated MLVA richness and prolonged

385 breakdown periods observed in beef fattening herds are associated with cattle purchases during

386 breakdowns. However, in other herd types, the accumulation of MLVA genotypes may result in

387 the absence of inwards cattle movements if herds are also exposed to infection from contiguous

388 farms, infectious wildlife, or a contaminated environment. Given the spatial structuring of the

389 M. bovis population (Skuce et al. 2010), we contend that it is more likely that re-infection from

390 local sources would present with same $M$. bovis strains that are already present in the herd and

391 local geographic area. Increasing MLVA richness would therefore have to be involved with the

392 introduction of MLVA types from over a larger geographical extent. Whilst there is some

393 evidence that badgers can occasionally travel long distances at scales of 7-20km (Byrne et al.

394 2014a), it may be less likely that long-distance badger movements are an important source of

395 MLVA richness relative to cattle movements which can traverse national scales (Brown et al.

396 2019). The increased resolution provided by pathogen whole-genome sequencing (WGS),

397 especially when more fully integrated with epidemiological data and modelling, may help to

398 better understand transmission dynamics and the relative role of hosts in a multi-host system

399 (Trewby et al. 2016).

400 
402 Karolemeas et al. 2010; Karolemeas et al. 2011; Olea-Popelka et al. 2008; Wolfe et al. 2010)

403 found that increasing herd size was positively associated with breakdowns lasting longer than 404365 days. This may be related to the inability to detect all bTB-positive animals using the non405 gold-standard ante-mortem SICCT test (Nuñez-Garcia et al. 2017). In NI, the relative sensitivity 406 of the SICCT test may be as low as $\sim 40 \%$ in chronically infected herds (Lahuerta-Marin et al. 407 2018). Undetected animals, where present, represent an ongoing reservoir of residual infection 408 which can lead to recrudescence of infection. The risk associated with herd size, however, may 409 also be confounded with production type. Here, we found that herds with a milk license (i.e. 410 dairy herds) were larger than herds without a milk license. Dairy farms may be associated with 411 particularly intensive production, potentially increasing within-herd transmission (i.e. 412 amplification) of infection (Alvarez et al. 2012; Menzies \& Neill 2000). Furthermore, there is 413 some evidence that the SICCT test performs poorly in dairy in NI settings compared to beef 414 (Lahuerta-Marin et al. 2018) which could exacerbate the problem presented by of residual 415 infection. In the final multivariable models presented here, however, the presence of a milk 416 license was not found to be an important predictor of breakdown duration. We hypothesize that 417 other variables which differ between production types (e.g. herd size) have captured some 418 important differences between animal husbandry practices which may be related to breakdown 419 duration. The number of reactors in the disclosing test was also positively associated with 422 breakdown duration. We speculate that the presence of a large number of reactors at the 423 disclosing test may indicate severity of infection, possibly arising from an environment which 
424 facilitates rapid within-herd transmission e.g. intensive farming units, or shared housing (Alvarez 425 et al. 2012). Unless all animals infected with M. bovis are identified and removed from the herd

426

427

428

429

430

431

432

433

434

435

436

437

438

439

440

441

442

443

444

445

446

\section{7}

as soon as possible, the rapid dissemination of infection will continue, thereby prolonging the outbreak duration (i.e. residual infection leading to within-herd recrudescence). Alternatively, many reactors at the disclosing test may indicate that infection has been either present or introduced since the preceding SICCT test, thereby providing a time period during which dissemination of infection to susceptible hosts within the herd could occur. We found that herd bTB history, measured by the presence of at least one previous breakdown in the study, was also associated with breakdown duration in the count model. Taken together, we hypothesise that a high number of disclosing reactors and a history of bTB indicates the presence of local infection (e.g. a contaminated environment, contiguous herds or infected wildlife), which may lead to increasingly prolonged outbreaks.

6

The presence of a lesioned animal at slaughter (LRS) was indicative of longer

breakdowns in our models, which is in line with previous findings (Doyle et al. 2016). We argue that the presence of a tuberculosis lesion is often evidence of undetected bTB infection within the herd (Olea-Popelka et al. 2008). Indeed, previous work from NI confirmed that $97 \%$ of

41 lesions from LRS animals were confirmed as bTB with histopathology or culture (Byrne et al. 2017). The relationship between bTB breakdown length and the presence of associated herds and elevated patch prevalence (Clegg et al. 2018; Doyle et al. 2016) illustrate the risk of infection from the local sources. Here, infection may originate from a shared contaminated environment (e.g. housing or grazing), which could lead to prolonged breakdowns if associated herds also contained infected animals. It may also point to shared use of equipment, or the 
447 spreading of contaminated slurry across multiple farms (O'Hagan et al. 2016). The positive

448 relationship between local geography and prolonged breakdowns identified here has been

449 observed previously in GB and the ROI (Olea-Popelka et al. 2004; Reilly \& Courtenay 2007).

450 We suggest that geographical location variables (DVO and patch) are also a proxy for highly

451 localised factors which could potentially influence breakdown length via exposure to other

452 infected hosts in the area. These include degree of farm fragmentation, conacre use (shared

453 grazing practice), and opportunities for contact with neighboring cattle ( O'Hagan et al. 2016;

454 White et al. 2013).

455

456

Wildlife and breakdown duration

457

In the univariable context, we identified a general positive relationship between

458 breakdown duration and main sett density. Unsurprisingly, in the multivariate context, main sett

459 density was confounded with other spatial and local variables (i.e. DVO and patch), making

460 inferences on the contribution of badger density to infection prolongation less clear. It is not yet

461 possible to conclusively distinguish between local sources of infection (which may include

462 wildlife, contiguous herds and environmental contamination), but given our data, we cannot

463 reject the hypothesis that badgers may be involved in the maintenance of local patch bTB

464 prevalence via spillback infection to cattle. Whether infected badger presence has a greater risk

465 of sporadic introduction of infection into herds (singular badger-cattle spillover), than longer-

466 term maintenance within herds (explosive introduction of infection elevated with cattle-cattle

467 transmission), remains to be determined. Whilst this study was unable to conclusively clarify the

468 relationship between badger density and breakdown duration, our data nevertheless reveal

469 important features that warrant further investigation in future studies. 
470 Thus, despite DVO capturing the risk associated with main sett density and the general positive

471 association between breakdown length and main sett density, there was some evidence of within-

472 DVO effects. Within five DVO areas (Ballymena, Coleraine, Dungannon, Larne and

473 Derry/Londonderry), increasing sett density was generally associated with longer breakdowns.

474 In the other five DVO areas (Armagh, Enniskillen, Newry, Newtownards and Omagh),

475 increasing sett density was generally associated with shorter breakdowns. The five DVOs with a 476 positive association between sett density and breakdown duration were areas of generally lower

477 badger sett densities (Reid et al. 2012). The DVOs with a negative association between sett

478 density and breakdown duration were generally associated with higher badger sett densities.

479 Whilst the interpretation of this is not straightforward, differences in farming practice (e.g. farm

480 fragmentation) or differences in badger ecology (e.g. population context dependent badger

481 dispersal; Byrne et al. 2019) across the region could partially explain this observation. However,

482 this does not preclude that the relationship between herd bTB risk from badgers may not be

483 simply be dependent on wildlife density; the sett density data provides no insight regarding

484 disease prevalence within the badger population. A spatially explicit model of disease

485 prevalence in badgers may resolve this in future. Indeed, future research could investigate

486 variation in wildlife TB transmission risk (LaHue et al. 2016) as a function of infection

487 prevalence as well as density, and investigate how that could help to partially explain patterns

488 within cattle data.

489

490

Conversely, infection risk in cattle may not be linked to badger disease prevalence or

491 population density, but may instead related to the relative frequency of interactions between

492 infected badgers and susceptible cattle (Böhm et al. 2009). Alternatively, it may be that indirect 
493 transmission of bTB via, for example, cattle accessing badger latrines, is more critically

494 associated with chronic bTB breakdowns as opposed to wildlife population density per se

495 (Campbell et al. 2019; Drewe et al. 2013). Furthermore, despite sett density being a convenient

496 metric, we must be careful when inferring the relationship between sett density and population

497 density, as the magnitude of the association can change depending on the local dynamics. For

498 example, population density can increase without necessarily an increase in setts via an increase

499 in the mean group size (Judge et al. 2014). Alternatively, where badger population densities are

500 depressed (e.g. though hunting, culling, or illegal disturbance), sett density metrics can

501 overestimate true local density. In Ireland, sett density was found to be good predictor of

502 increased herd breakdown risk early in a six-year study, but progressively became a weaker

503 predictor as a program of targeted badger culling reduced population density (Byrne et al.

504 2014b). Therefore, investigating intricate relationships between wildlife and domestic hosts may

505 well require even more detailed information around population abundance at large scales, in the

506 Northern Ireland context this could include mark-recapture and/or the use of remote camera

507 trapping technologies (Campbell et al. 2019).

508

509 Conclusions

510 The most important predictor of breakdown duration in our models was elevated MLVA

511 genotype richness, which is often a feature of beef fattening herds and linked to the practice of

512 purchasing cattle from over a wide geographic extent. We conclude that in at least some specific

513 herds, prolonged restriction periods may primarily be a product of inwards cattle movements

514 during a breakdown. For all other herd types, our results support the hypothesis that breakdown

515 duration is principally a function of the inability to eradicate residual infection already present 
516 within the herd, and/or repeated infection from the local environ. In many instances, failure to

517 clear residual infection may be related to the poor performance of the ante-mortem diagnostic

518 SICCT test, which permits the retention of infected animals. Our data suggest that infected

519 wildlife (captured by sett density), contiguous herds (captured by patch prevalence and

520 associated herds) and a contaminated environment (also captured by patch prevalence) all likely

521 contribute to varying extents to protracted breakdowns. However, given that it is not yet

522 possible to positively distinguish between these various infection routes, determining the relative

523 contribution each potential source was beyond the scope of this study. We posit that badgers

524 may be involved in prolonging bTB breakdowns via spillback infection into the cattle

525 population, supplemented with cattle-to-cattle transmission (amplification) once infection is

526 introduced to the herd. However, the general positive association between badger sett density

527 and breakdown duration may not simply be a function of badger population density, and could

528 also be product of density-dependent badger behavior which may possibly influence contact rates

529 between badgers and cattle.

530

531 Acknowledgements

532

We would like to thank the staff in the Agri-Food and Biosciences Institute Veterinary

533 Sciences Division (VSD) who contributed to the bTB strain-typing work.

534

535

References

536

537

538

539

540

541

542

Abernethy DA, Denny GO, Menzies FD, McGuckian P, Honhold N, and Roberts AR. 2006. The Northern Ireland programme for the control and eradication of Mycobacterium bovis. Veterinary Microbiology 112:231-237. 10.1016/j.vetmic.2005.11.023

Abernethy DA, Upton P, Higgins IM, McGrath G, Goodchild AV, Rolfe SJ, Broughan JM, Downs SH, Clifton-Hadley R, Menzies FD, de la Rua-Domenech R, Blissitt MJ, 
543

544

545

546

547

548

549

550

551

552

553

554

555

556

557

558

559

560

561

562

563

564

565

566

567

568

569

570

571

572

573

574

575

576

577

578

579

580

581

582

583

584

585

586
Duignan A, and More SJ. 2013. Bovine tuberculosis trends in the UK and the Republic of Ireland, 1995-2010. Veterinary Record 172:312-312.

10.1136/vr.100969

AHVLA. 2016. Bovine tuberculosis: Infection status in cattle in GB. Annual surveillance report for the period January to December 2015.

Allen AR, Skuce RA, and Byrne AW. 2018. Bovine Tuberculosis in Britain and Ireland A Perfect Storm? the Confluence of Potential Ecological and Epidemiological Impediments to Controlling a Chronic Infectious Disease. Frontiers in Veterinary Science 5. 10.3389/fvets.2018.00109

Brown, E., Marshall, A.H., Mitchell, H.J. and Byrne, A.W., 2019. Cattle movements in Northern Ireland form a robust network: implications for disease management. Preventive Veterinary Medicine, 170, p.104740.

Byrne, AW, Graham J, Brown C, Donaghy A, Guelbenzu-Gonzalo M, McNair J, Skuce R, Allen A, and McDowell S. 2017. Bovine tuberculosis visible lesions in cattle culled during herd breakdowns: the effects of individual characteristics, trade movement and co-infection. BMC Veterinary Research 13:400. 10.1186/s12917017-1321-z

Byrne, A.W., O'Keeffe, J., Buesching, C.D. and Newman, C., 2019. Push and pull factors driving movement in a social mammal: context dependent behavioural plasticity at the landscape scale. Current Zoology. 65, 5, 517-525 https://doi.org/10.1093/cz/zoy081

Byrne, A.W., Quinn, J.L., O'Keeffe, J.J., Green, S., Paddy Sleeman, D., Wayne Martin, S. and Davenport, J., 2014. Large-scale movements in E uropean badgers: has the tail of the movement kernel been underestimated?. Journal of Animal Ecology, 83(4), pp.991-1001.

Byrne, A.W., White, P.W., McGrath, G., James, O. and Martin, S.W., 2014. Risk of tuberculosis cattle herd breakdowns in Ireland: effects of badger culling effort, density and historic large-scale interventions. Veterinary Research, 45(1), p.109.

Clegg TA, Good M, Hayes M, Duignan A, McGrath G, and More SJ. 2018. Trends and Predictors of Large Tuberculosis Episodes in Cattle Herds in Ireland. Frontiers in Veterinary Science 5:86.

DAERA. 2017. Tuberculosis Staff Instructions. In: Department of Agriculture EaRAVS, editor. Belfast: Department of Agriculture, Environment and Rural Affairs Veterinary Service

DAERA. 2018. Tuberculosis disease statistics in Northern Ireland 2018. Available at https://www.daera-ni.gov.uk/publications/tuberculosis-disease-statistics-northernireland-2018.

Doyle LP, Courcier EA, Gordon AW, O’Hagan MJH, and Menzies FD. 2016. Bovine tuberculosis in Northern Ireland: Risk factors associated with duration and recurrence of chronic herd breakdowns. Prev Vet Med 131:1-7.

10.1016/j.prevetmed.2016.06.016

Gallagher J, and Clifton-Hadley RS. 2000. Tuberculosis in badgers; a review of the disease and its significance for other animals. Research in Veterinary Science 69:203-217. 10.1053/rvsc.2000.0422 
587

588

589

590

591

592

593

594

595

596

597

598

599

600

601

602

603

604

605

606

607

608

609

610

611

612

613

614

615

616

617

618

619

620

621

622

623

624

625

626

627

628

629

630
Gallagher MJ, Higgins IM, Clegg TA, Williams DH, and More SJ. 2013. Comparison of bovine tuberculosis recurrence in Irish herds between 1998 and 2008. Prev Vet Med 111:237-244. 10.1016/j.prevetmed.2013.05.004

Griffin JM, Hahesy T, Lynch K, Salman MD, McCarthy J, and Hurley T. 1993. The association of cattle husbandry practices, environmental factors and farmer characteristics with the occurence of chronic bovine tuberculosis in dairy herds in the Republic of Ireland. Prev Vet Med 17:145-160. https://doi.org/10.1016/01675877(93)90025-O

Houtsma E, Clegg TA, Good M, and More SJ. 2018. Further improvement in the control of bovine tuberculosis recurrence in Ireland. Veterinary Record 183:622-622. $10.1136 / v r .104642$

Humblet M-F, Boschiroli ML, and Saegerman C. 2009. Classification of worldwide bovine tuberculosis risk factors in cattle: a stratified approach. Veterinary Research 40:50. 10.1051/vetres/2009033

Karolemeas K, Donnelly CA, Conlan AJK, Mitchell AP, Clifton-Hadley RS, Upton P, Wood JLN, and McKinley TJ. 2012. The effect of badger culling on breakdown prolongation and recurrence of bovine tuberculosis in cattle herds in Great Britain. PLOS ONE 7:e51342. 10.1371/journal.pone.0051342

Karolemeas K, Mckinley TJ, Clifton-Hadley RS, Goodchild AV, Mitchell A, Johnston WT, Conlan AJK, Donnelly CA, and Wood JLN. 2010. Predicting prolonged bovine tuberculosis breakdowns in Great Britain as an aid to control. Prev Vet Med 97:183-190. 10.1016/j.prevetmed.2010.09.007

Karolemeas K, Mckinley TJ, Clifton-Hadley RS, Goodchild AV, Mitchell A, Johnston WT, Conlan AJK, Donnelly CA, and Wood JLN. 2011. Recurrence of bovine tuberculosis breakdowns in Great Britain: Risk factors and prediction. Prev Vet Med 102:22-29. 10.1016/j.prevetmed.2011.06.004

Lahuerta-Marin A, Gallagher M, McBride S, Skuce R, Menzies F, McNair J, McDowell SWJ, and Byrne AW. 2015. Should they stay, or should they go? Relative future risk of bovine tuberculosis for interferon-gamma test-positive cattle left on farms. Veterinary Research 46:90. 10.1186/s13567-015-0242-8

Milne GM, Graham J, Allen A, Lahuerta-Marin A, McCormick C, Presho E, Skuce R, and Byrne AW. 2019. Spatiotemporal analysis of prolonged and recurrent bovine tuberculosis breakdowns in Northern Irish cattle herds reveals a new infection hotspot. Spatial and Spatio-temporal Epidemiology 28:33-42. https://doi.org/10.1016/j.sste.2018.11.002

More SJ, Houtsma E, Doyle L, McGrath G, Clegg TA, de la Rua-Domenech R, Duignan A, Blissitt MJ, Dunlop M, Schroeder PG, Pike R, and Upton P. 2018. Further description of bovine tuberculosis trends in the United Kingdom and the Republic of Ireland, 2003-2015. The Veterinary Record 183:717-717. 10.1136/vr.104718

NIAO. 2018. The control of Bovine Tuberculosis in Northern Ireland. Northern Ireland Audit Office.

Reid N, Etherington TR, Wilson GJ, Montgomery WI, and McDonald RA. 2012. Monitoring and population estimation of the European badger Meles meles in Northern Ireland. Wildlife Biology 18:46-57. 
631 Reilly LA, and Courtenay O. 2007. Husbandry practices, badger sett density and habitat composition as risk factors for transient and persistent bovine tuberculosis on UK cattle farms. Prev Vet Med 80:129-142. 10.1016/j.prevetmed.2007.02.002

Robinson PA. 2017. Farmers and bovine tuberculosis: Contextualising statutory disease control within everyday farming lives. Journal of Rural Studies 55:168-180. 10.1016/j.jurstud.2017.08.009

Skuce RA, Mallon TR, McCormick CM, McBride SH, Clarke G, Thompson A, Couzens C, Gordon AW, and McDowell SWJ. 2010. Mycobacterium bovis genotypes in Northern Ireland: herd-level surveillance (2003 to 2008). Veterinary Record 167:684-689. 10.1136/vr.c5108

Wolfe DM, Berke O, Kelton DF, White PW, More SJ, O'Keeffe J, and Martin SW. 2010. From explanation to prediction: A model for recurrent bovine tuberculosis in Irish 644 cattle herds. Prev Vet Med 94:170-177. 10.1016/j.prevetmed.2010.02.010 


\section{Table 1 (on next page)}

Summary statistics of the fixed effect explanatory variables. 
1 Table 1: Summary statistics of the fixed effect explanatory variables.

\begin{tabular}{|c|c|c|c|c|}
\hline Variable & Count & Median & Mean & IQR (1st-3rd) \\
\hline breakdown_length & & 188 & 225.6 & $140-260$ \\
\hline herd_size & & 93 & 141.2 & $42-190$ \\
\hline outbreak_reactors & & 1 & 2.84 & $1-2$ \\
\hline total_reactors & & 3 & 7.66 & $2-8$ \\
\hline year_patch_prev & & 8.85 & 9.89 & $6.01-12.55$ \\
\hline mean_patch_prev & & 10.18 & 10.73 & $7.83-13.24$ \\
\hline MLVA_richness & & 1 & 1.25 & $1-1$ \\
\hline main_sett & & 0.74 & 0.77 & $0.56-0.92$ \\
\hline outwards_moves_year_before & & 52 & 98.22 & $23-106$ \\
\hline inwards_moves_year_be & & 9 & 59.78 & $1-42$ \\
\hline LRS & 2209 & & & \\
\hline milk_licence & 2360 & & & \\
\hline associated_herds & 1501 & & & \\
\hline previous_breakdown & 2061 & & & \\
\hline herd_type beef & 3617 & & & \\
\hline herd_type dairy & 2275 & & & \\
\hline herd_type other & 98 & & & \\
\hline
\end{tabular}




\section{Table 2 (on next page)}

Parameter estimates of the fixed effectexplanatory variables in the final model for both the count model (negative binomial) and ordinal model . 
1 Table 2: Parameter estimates of the fixed effect explanatory variables in the final model for both

2 the count model (negative binomial) and ordinal model.

3

\begin{tabular}{|c|c|c|c|c|c|c|}
\hline Variable & IRR & $\begin{array}{l}95 \% \text { CI } \\
\text { Lower }\end{array}$ & $\begin{array}{c}\text { 95\%CI } \\
\text { Upper }\end{array}$ & OR & $\begin{array}{l}95 \% \text { CI } \\
\text { Lower }\end{array}$ & $\begin{array}{l}95 \% \text { CI } \\
\text { Upper }\end{array}$ \\
\hline log(herd_size $)$ & 1.05 & 1.04 & 1.06 & 1.26 & 1.20 & 1.32 \\
\hline $\log$ (outbreak_reactors) & 1.05 & 1.04 & 1.06 & 1.34 & 1.26 & 1.43 \\
\hline $\log ($ mean_patch_prev $)$ & 1.04 & 1.01 & 1.07 & 1.20 & 1.04 & 1.37 \\
\hline log(MLVA_Richness) & 1.62 & 1.58 & 1.67 & 7.06 & 6.04 & 8.24 \\
\hline LRS_binary 1 & 1.12 & 1.09 & 1.14 & 1.79 & 1.59 & 2.01 \\
\hline associated_herds_binary1 & 1.10 & 1.07 & 1.13 & 1.49 & 1.32 & 1.69 \\
\hline previous_breakdown & 1.04 & 1.02 & 1.07 & - & - & - \\
\hline
\end{tabular}

4

5 


\section{Table 3 (on next page)}

Parameter estimates of the explanatory variablesin an alternative count model, allowing main sett to vary on a per DVO basis. 
1 Table 3: Parameter estimates of the explanatory variables in an alternative count model,

2 allowing main sett to vary on a per DVO basis.

3

\begin{tabular}{lcc} 
DVO & $\begin{array}{c}\text { main_sett } \\
\text { slope }\end{array}$ & Intercept \\
\hline Armagh & -0.030 & 5.031 \\
Ballymena & 0.039 & 4.874 \\
Coleraine & -0.004 & 4.971 \\
Dungannon & -0.031 & 5.033 \\
Enniskillen & 0.047 & 4.856 \\
Larne & 0.017 & 4.923 \\
Londonderry & 0.031 & 4.892 \\
Newry & -0.107 & 5.204 \\
Newtownards & -0.010 & 4.986 \\
Omagh & 0.022 & 4.913
\end{tabular}

\begin{tabular}{lcccccc} 
Variable & Est & Std. Error & z & IRR & $\begin{array}{c}\text { 95\%CI } \\
\text { Lower }\end{array}$ & $\begin{array}{c}\text { 95\%CI } \\
\text { Upper }\end{array}$ \\
\hline Intercept & 4.96 & 0.04 & 120.1 & 143 & 131.89 & 155.04 \\
$\log ($ herd_size) & 0.05 & 0.00 & 9.80 & 1.05 & 1.04 & 1.06 \\
$\log$ (outbreak_reactors) & 0.05 & 0.01 & 7.57 & 1.05 & 1.04 & 1.06 \\
log(main_sett) & 0.01 & 0.02 & 0.39 & 1.01 & 0.97 & 1.05 \\
log(MLVA_Richness) & 0.48 & 0.01 & 34.51 & 1.62 & 1.58 & 1.67 \\
LRS_binary1 & 0.11 & 0.01 & 9.68 & 1.12 & 1.09 & 1.14 \\
associated_herds_binary1 & 0.09 & 0.01 & 7.21 & 1.10 & 1.07 & 1.12 \\
previous_breakdown & 0.04 & 0.01 & 3.57 & 1.04 & 1.02 & 1.07
\end{tabular}

4 
Figure 1

The distribution of continuous variables across each DVO area within Northern Ireland.

A number of variables exhibited little variation in the median values per-DVO and are therefore not displayed (outbreak reactors, median = 1 for all DVOs; MLVA Richness, median $=1$ for all DVOs). 

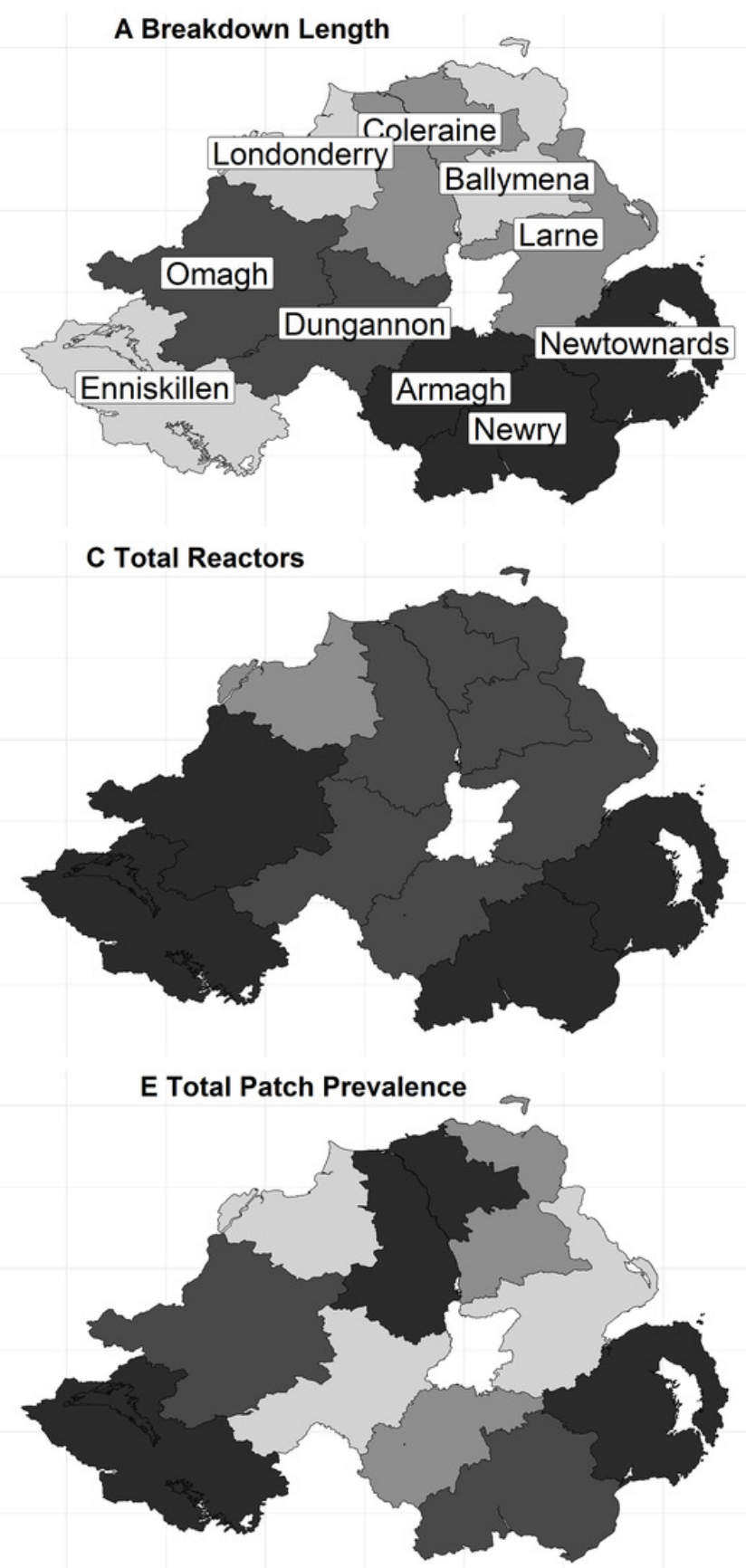

G Out-moves Before Breakdown

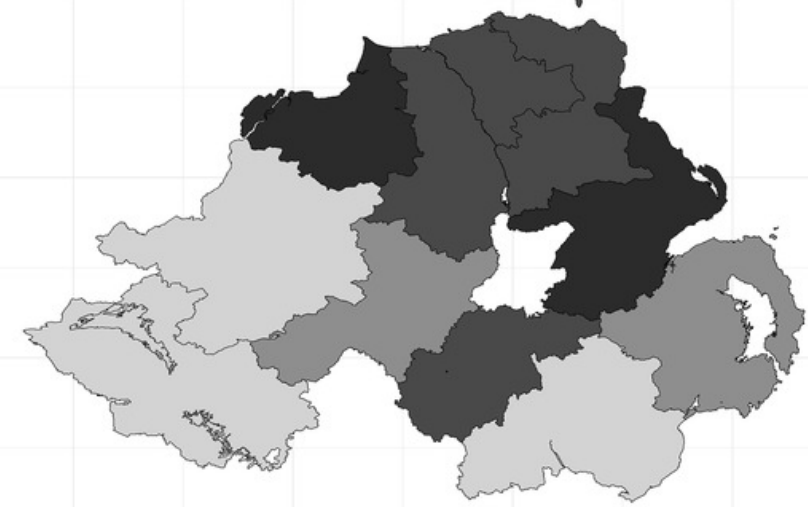

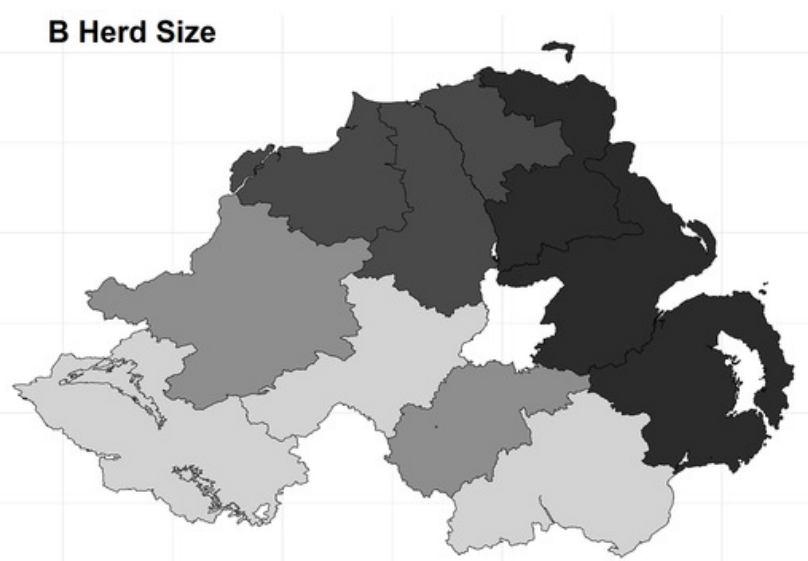

D Yearly Patch Prevalence

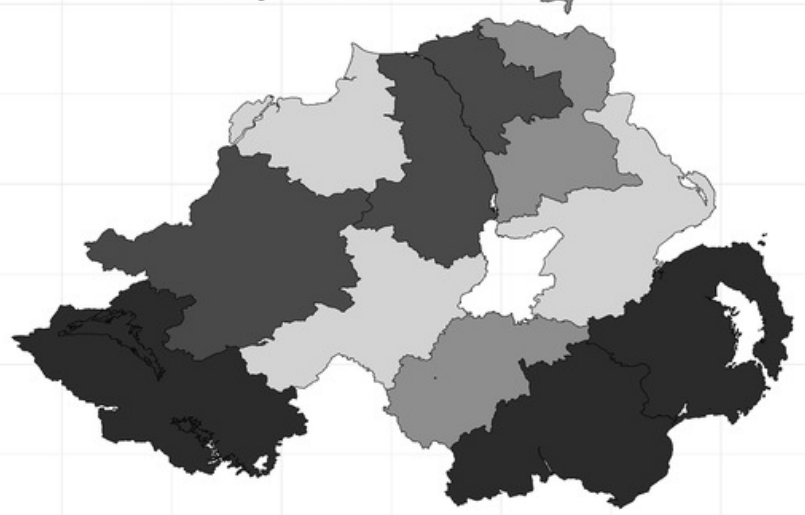

F Main Sett Density

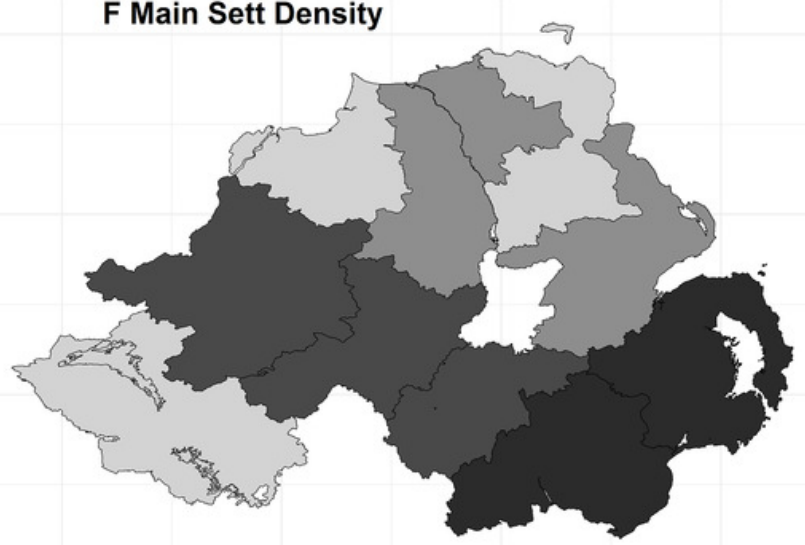

H In-moves Before Breakdown

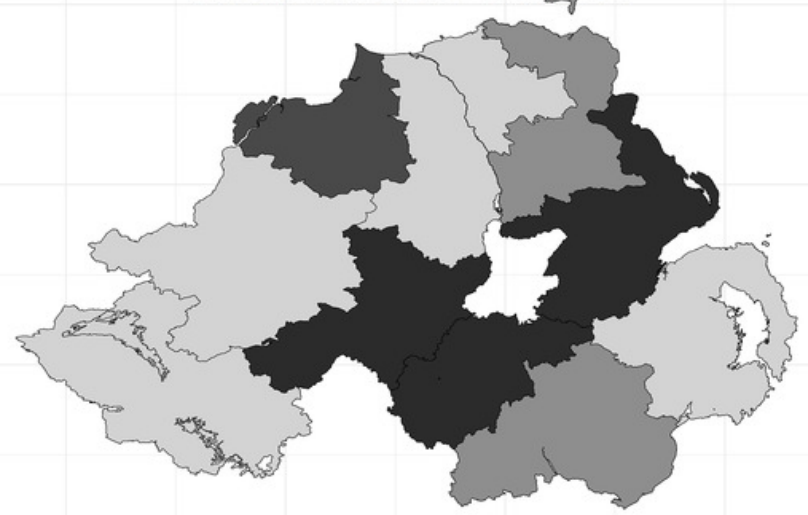


Figure 2

Distribution of breakdown length
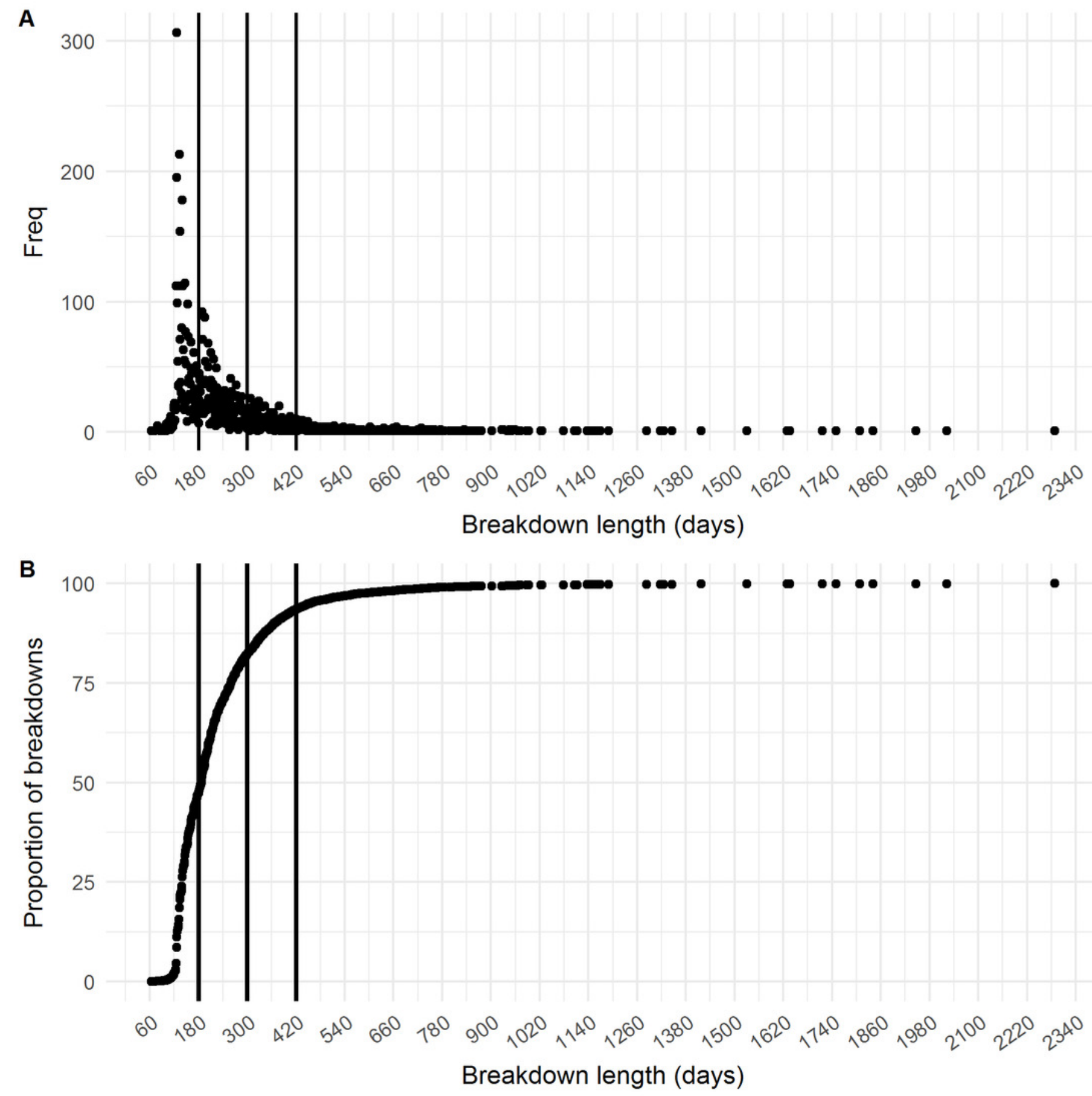


\section{Figure 3}

\section{Comparison of parameter estimates across models}

Comparison of parameter estimates for the six explanatory variables obtained from the ordinal regression model with four categories (full model), compared to parameter estimates obtained from three binary logistic regression models (model type). 


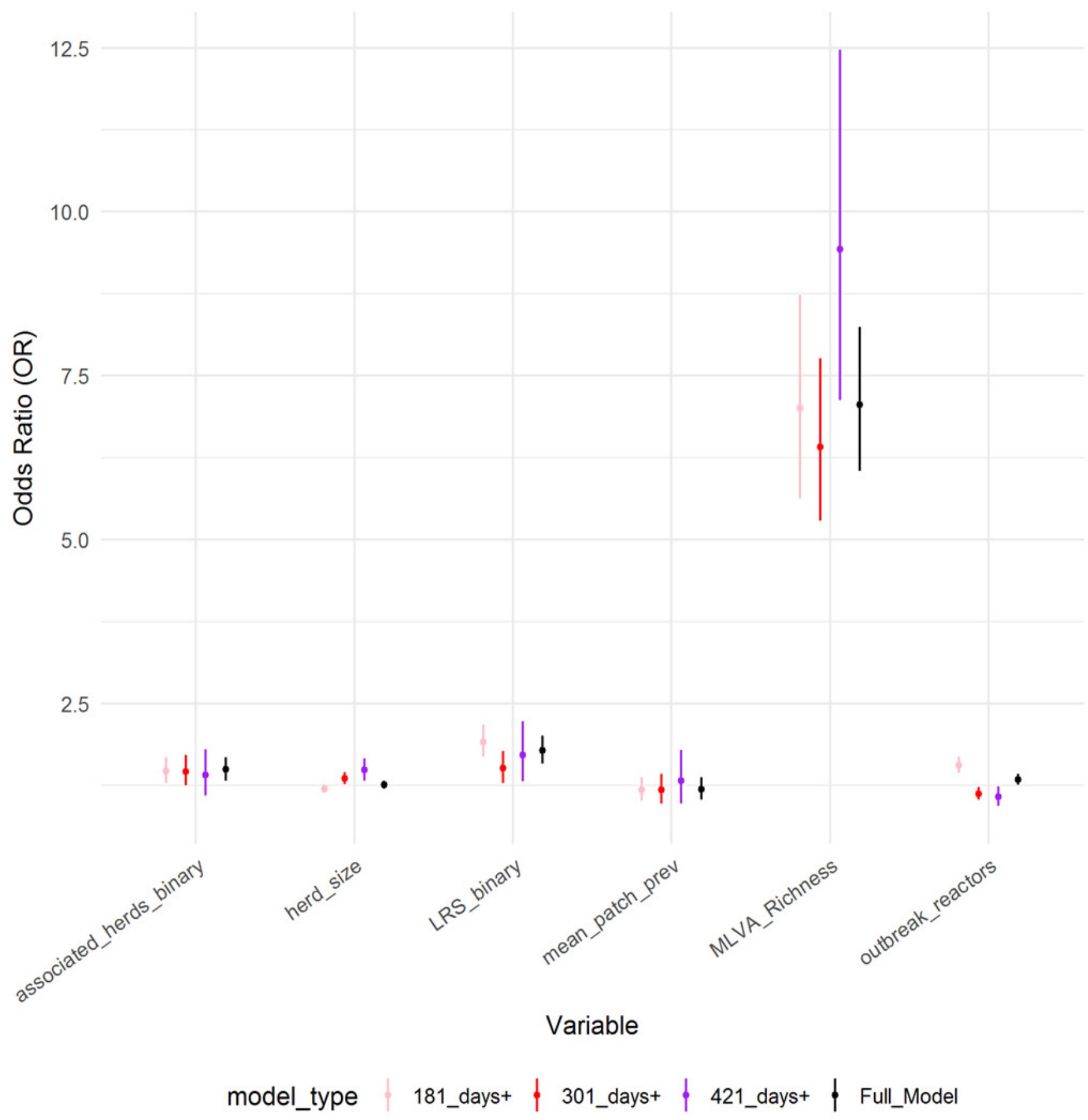


Figure 4

Relationship between (A) MLVA genotype richnessand categorical breakdown duration; (B) how the number of reactors over abreakdown differs between production types; (C) how the breakdown lengthdiffers between production types and; (D) the confounding betwe 


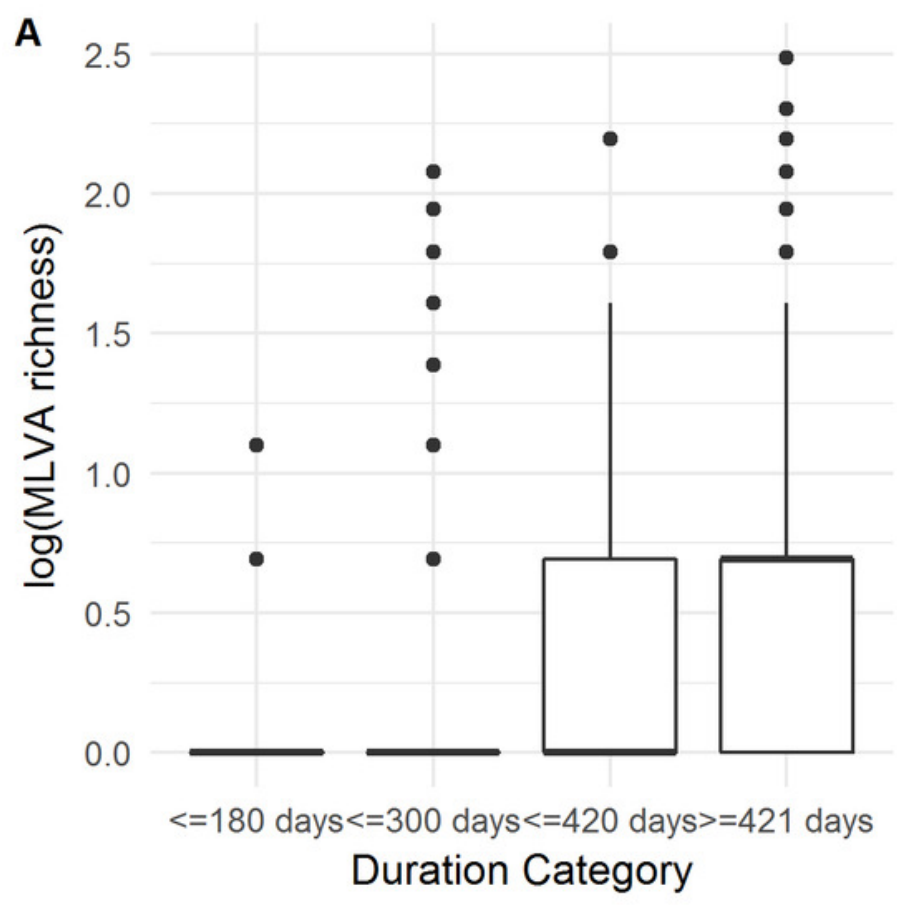

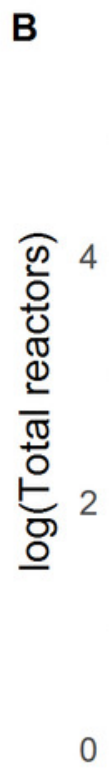

0

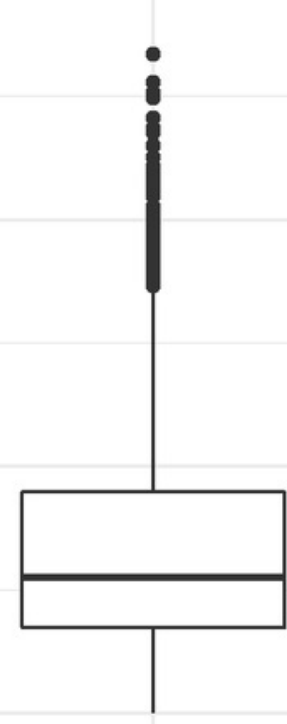

0

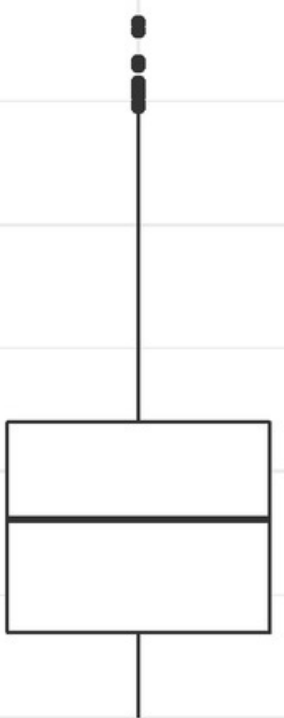

1

Presence(1)/Absence( 0 ) of milk licence

C
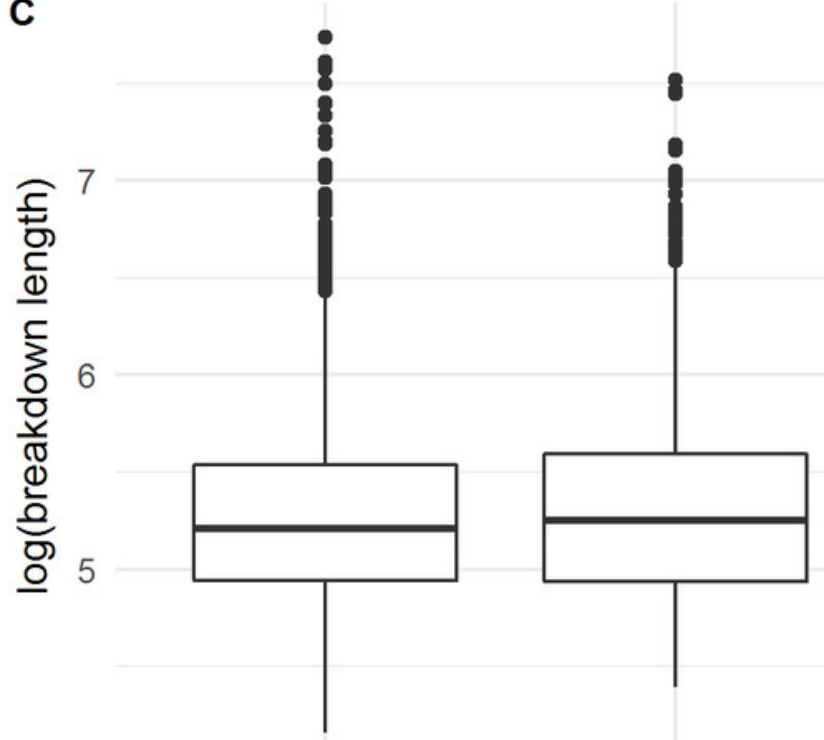

4

0

1

Presence(1)/Absence( 0 ) of milk licence

D

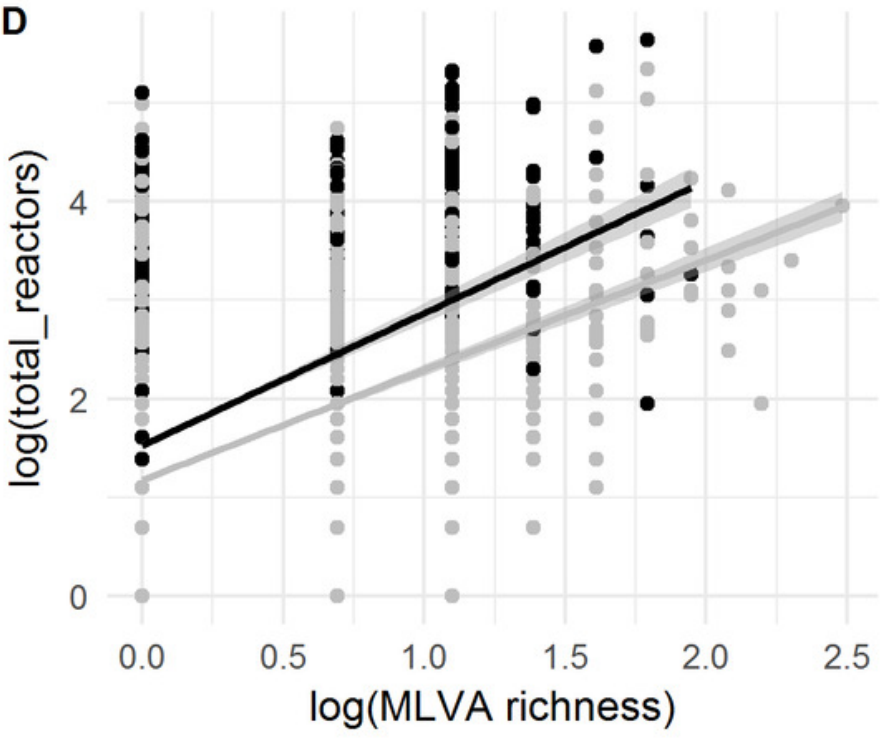

milk_licence_binary $\rightarrow 0 \rightarrow 1$ 
Figure 5

\section{Correlations}
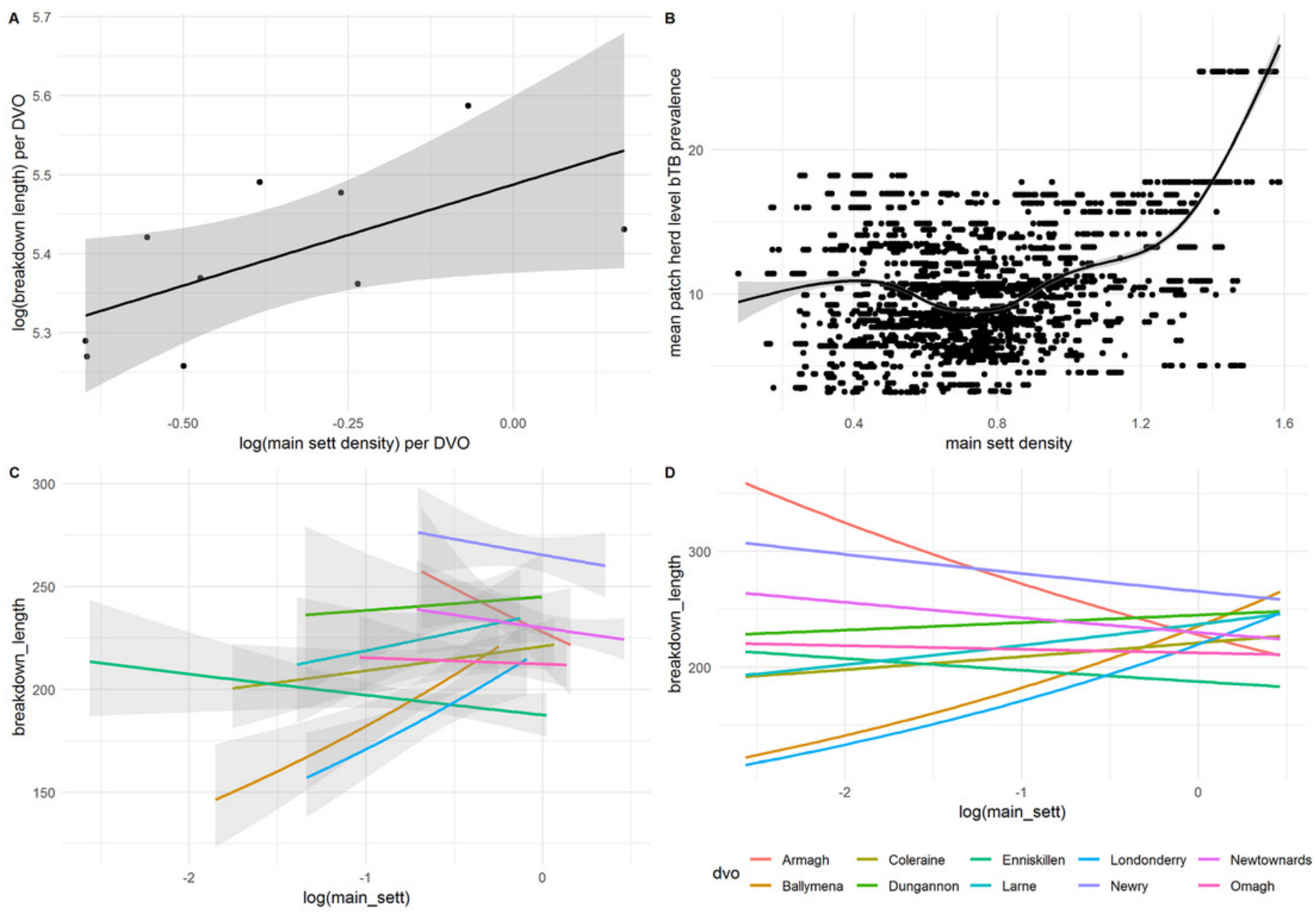